\title{
Correction to the article An étalé space construction for stacks
}

DAVID CARCHEDI

The definition of a category of spaces given in An étalé space construction for stacks is problematic in that the main examples do not satisfy the axioms listed there. In this erratum, we give an alternative definition which encompasses our main examples and for which the results and proofs of this paper still hold.

22A22, 58H05; $18 \mathrm{~B} 25$

\section{Introduction}

The results and arguments in [1] are meant to apply to various classes of spaces, including the following main examples:

(I) Locales.

(II) Sober topological spaces.

(III) Any type of manifold; eg smooth manifolds, $C^{k}$ manifolds, analytic manifolds, complex manifolds, super manifolds, etc.

(IV) Schemes (with the Zariski topology).

Remark 1.1 We do not require the underlying topological space of a manifold to be $2^{\text {nd }}$-countable or Hausdorff. Likewise, we do not impose any separation conditions on our schemes.

Remark 1.2 Recall that a topological space $X$ is sober [1, Definition A.6] if it can be reconstructed up to homeomorphism from its lattice of open subsets. A locale is essentially the concept of space that arises from the study of topologies as abstract lattices without the demand that these lattices can be embedded into the power set of some set. In particular, for a topological space $X$, its lattice of open subsets $\mathcal{O}(X)$ is a locale; see [1, Appendix A] for more details. 
Rather than repeating the analogous arguments for each of these categories, the paper simply takes $S$ to be a suitable category of spaces, together with a suitable collection of morphisms called the local homeomorphisms. For example, if $S$ were taken to be smooth manifolds, the word space would mean smooth manifold, and the phrase local homeomorphism would mean local diffeomorphism. Any such category comes equipped with a canonical functor

$$
U: S \rightarrow \mathbb{L O C}
$$

to the category of locales.

It is easy to check that the only properties of such a category of spaces that were used in any of the proofs are:

(1) Pullbacks against local homeomorphisms exist.

(2) $S$ is closed under taking open subspaces or more generally:

(3) If $h: T \rightarrow U X$ is a local homeomorphism of locales, then $h=U(f)$ for $f: Z \rightarrow X$ a local homeomorphism in $S$.

(4) If $S^{\text {et }}$ denotes the category spanned by the local homeomorphisms of $S$, then for any space $X$ of $S$, the category $S^{\text {et }} X$ is canonically equivalent to the category of sheaves on the underlying space (or locale) $U X$ of $X$ (and is hence a topos).

(5) $S$ has coproducts.

[1, Appendix C] lists certain axioms for a category $S$ equipped with a subcategory $S^{\text {et }}$ of local homeomorphisms and a functor $U: S \rightarrow \mathbb{L O C}$ to satisfy for the results of the paper to apply to it. Unfortunately, there are a couple of problems with the axioms listed. The least serious problem is that condition (2) of [1, Definition B.1] should instead read:

If $f$ and $g$ are arrows in $S$ such that $g f$ exists, then if $f$ and $g$ are in $S^{\text {et }}$, so is $g f$. If instead $g f$ and $g$ are in $S^{\mathrm{et}}$, so is $f$.

However, anyone familiar with the behavior of local homeomorphisms would probably spot and fix this error themselves. The more serious error is in [1, Definition B.2] which defines category of spaces. The problem is condition (b) of this definition, which states that if $T$ is a space in the category $S, U T$ is its underlying locale, and $\varphi: U T \rightarrow X$ is a surjective local homeomorphism, then there exists another space $Z$ in $S$ such that $U Z \cong X$. It is then claimed that the category of smooth manifolds satisfies this condition. However, this is clearly false by the existence of topological manifolds which admit no smooth atlas. 


\section{A replacement for [1, Appendix C]}

We now propose a different definition for what a category of spaces should be, which satisfies conditions (1)-(5) needed for the arguments of [1] to go through and include all the examples (I)-(IV). We start by introducing the concept of a ringed locale.

Definition 2.1 Fix a commutative ring $k$. A ringed locale over $k$ is a locale $\lambda$ together with a sheaf $\mathscr{A}_{\lambda}$ of commutative $k$-algebras on $\lambda$. A map of ringed locales

$$
\left(\lambda, \mathscr{A}_{\lambda}\right) \rightarrow\left(\lambda^{\prime}, \mathscr{A}_{\lambda}^{\prime}\right)
$$

consists of a map of locales $f: \lambda \rightarrow \lambda^{\prime}$ and a morphism of sheaves $\alpha: f^{*} \mathscr{A}_{\lambda}^{\prime} \rightarrow \mathscr{A}_{\lambda}$. Denote by $\mathcal{R} \mathcal{L}_{k}$ the category of ringed locales over $k$. Denote by $\mathcal{R S}_{k}$ the full subcategory consisting of those locally ringed locales $\left(\lambda, A_{\lambda}\right)$ for which $\lambda$ has enough points.

Remark 2.2 By stone duality [1, Theorem A.1], $\mathcal{R} \mathcal{S}_{k}$ is equivalent to the full subcategory of the classical category of ringed spaces over $k$, spanned by those ringed spaces whose underlying topological space is sober.

Associated to a ringed locale $\left(\lambda, \mathscr{A}_{\lambda}\right)$ one gets a ringed space $\left(\operatorname{pt}(\lambda), \eta_{\lambda}^{*}\left(\mathscr{A}_{\lambda}\right)\right)$ where $\eta$ is the unit of the adjunction $\mathcal{O} \dashv$ pt of [1, Theorem A.1]. This induces a functor $p: \mathcal{R} \mathcal{L}_{k} \rightarrow \mathcal{R S}_{k}$ between the category of ringed locales and the category of ringed (sober) spaces over $k$; see Remark 2.5.

Definition 2.3 A locally ringed locale over a commutative ring $k$ is a ringed locale

$$
\left(\lambda, A_{\lambda}\right)
$$

over $k$ such that $p\left(\left(\lambda, \mathscr{A}_{\lambda}\right)\right)$ is a locally ringed space, ie a ringed locale over $k\left(\lambda, \mathscr{A}_{\lambda}\right)$ such that the stalks of $\mathscr{A}_{\lambda}$ over each point of $\lambda$ in the sense of [1, Definition A.4] is a local ring. A morphism of locally ringed locales is a map

$$
(f, \alpha):\left(\lambda, \mathscr{A}_{\lambda}\right) \rightarrow\left(\lambda^{\prime}, \mathscr{A}_{\lambda}^{\prime}\right)
$$

of ringed locales over $k$ such that $p((f, \alpha))$ is a map of locally ringed spaces. Denote by $\mathcal{L} \mathcal{R} \mathcal{L}_{k}$ the category of locally ringed locales.

Definition 2.4 A morphism $\left(\lambda, \mathscr{A}_{\lambda}\right) \rightarrow\left(\lambda^{\prime}, \mathscr{A}_{\lambda}^{\prime}\right)$ in $\mathcal{L} \mathcal{R} \mathcal{L}_{k}$ is a local homeomorphism if the underlying map of locales $f: \lambda \rightarrow \lambda^{\prime}$ is a local homeomorphism in the sense of [1, Definition A.11] and the map $f^{*} \mathscr{A}_{\lambda}^{\prime} \rightarrow \mathscr{A}_{\lambda}$ is an isomorphism. Such a map is an open embedding if additionally the map $\lambda \rightarrow \lambda^{\prime}$ is an open embedding of locales in the sense of [1, Definition A.9]. 
For example, if $M$ and $N$ are smooth manifolds, local homeomorphisms

$$
\left(M, C_{M}^{\infty}\right) \rightarrow\left(N, C_{N}^{\infty}\right)
$$

over $\mathbb{R}$ are the same as local diffeomorphisms, and open embeddings are the same as smooth open embeddings.

Remark 2.5 Local homeomorphisms are stable under pullbacks in $\mathcal{L} \mathcal{R} \mathcal{L}_{k}$

Definition 2.6 By an open subspace of a locally ringed locale $\left(\lambda, \mathscr{A}_{\lambda}\right)$ we mean an open subset $U \subseteq \lambda$ (in the sense of [1, Definition A.8]) equipped with the pullback structure sheaf $\left(U,\left.A_{\lambda}\right|_{U}\right)$. An open covering family of a locally ringed locale $\left(\lambda, A_{\lambda}\right)$ is a collection of open embeddings

$$
\left(\left(U_{\alpha}, \mathscr{A}_{U_{\alpha}}\right) \rightarrow\left(\lambda, \mathscr{A}_{\lambda}\right)\right)_{\alpha}
$$

such that the underlying collection of open embeddings of locales

$$
\left(U_{\alpha} \rightarrow \lambda\right)_{\alpha}
$$

is an open covering in the sense of [1, Definition A.10].

Let us fix a category $\tau$ to be either the category of locales $\mathbb{L} \mathbb{O C}$, the category of locally ringed locales $\mathcal{L} \mathcal{R} \mathcal{L}_{k}$ over a fixed commutative ring $k$, or the analogously defined category $\mathcal{S} \mathcal{L} \mathcal{R} \mathcal{L}_{A}$ of locales locally ringed in supercommutative $A$-algebras, where $A$ is a supercommutative ring.

Definition 2.7 A subcategory $S$ of $\tau$ will be called a category of spaces if the following two properties hold:

(i) If $X$ is in $S$ and $Y \rightarrow X$ is a local homeomorphism, then $Y$ is in $S$.

(ii) If $\left(U_{\alpha} \rightarrow X\right)$ is an open covering family of $X$ such that each $U_{\alpha}$ is in $S$, then $X$ is in $S$.

We will refer to objects of $S$ simply as spaces. A map in $S$ is a local homeomorphism if it is one in $\tau$. By $S^{\text {et }}$ we mean the category spanned by the objects of $S$ and their local homeomorphisms.

Note that a category of spaces comes canonically with a functor $U: S \rightarrow \mathbb{L O C}$ sending a space $X$ to its underlying locale. (When $\tau$ is $\mathbb{L O C}, U$ is just the inclusion of the subcategory $S$ ).

Condition (i) above has many immediate consequences. For example, combining (i) with Remark 2.5, we see that pullbacks against local homeomorphisms always exist in $S$. Condition (i) also implies that $S$ is closed under the taking of open subspaces. The following proposition is also a consequence of condition (i). 
Proposition 2.8 Let $X$ be a space in $S$. There is a canonical equivalence of categories

$$
S^{\mathrm{et}} / X \simeq \operatorname{Sh}(U X)
$$

Proof The étale space construction provides a canonical equivalence of categories $\operatorname{Sh}(U X) \simeq \operatorname{Et}(U X)$, where $\operatorname{Et}(U X)$ is the category of local homeomorphisms over $X$; see [1, Theorem A.2]. It therefore suffices to observe that the canonical functor

$$
S^{\mathrm{et}} / X \rightarrow \operatorname{Et}(U X)
$$

induced by $U$ is an equivalence. When $\tau=\mathbb{L} \mathbb{O C}$, this follows immediately from condition (i). When $\tau=\mathcal{L} \mathcal{R} \mathcal{L}_{k}$ or $\mathcal{S} \mathcal{L} \mathcal{R} \mathcal{L}_{A}$, the functor

$$
\operatorname{Et}(U X) \rightarrow S^{\mathrm{et}} / X
$$

which sends a local homeomorphism of locales $f: \lambda \rightarrow \lambda^{\prime}$ to $\left(\lambda^{\prime}, f^{*} \mathscr{A}_{\lambda}\right) \rightarrow\left(\lambda, \mathscr{A}_{\lambda}\right)$ is the desired inverse.

Condition (ii) also implies the existence of arbitrary coproducts, since they exist in $\tau$ and $\left(X_{j} \hookrightarrow \bigsqcup_{i} X_{i}\right)$ is an open covering family.

The following is a non-exhaustive list of examples of categories of spaces in the sense of Definition 2.7:

(I) Locales.

(II) Sober topological spaces.

(III) Any type of manifold (eg smooth manifolds, $C^{k}$ manifolds, analytic manifolds, complex manifolds, super manifolds, etc), provided we remove all separation conditions. For example, manifolds will neither be assumed paracompact nor Hausdorff.

(IV) Schemes over a commutative ring $k$. Again, we do not impose any separation conditions.

Definition 2.9 We say a collection of local homeomorphisms $\left(T_{\alpha} \rightarrow T\right)_{\alpha}$ in $S$ is a covering family of local homeomorphisms if the induced morphism

$$
\coprod_{\alpha} U T_{\alpha} \rightarrow U T
$$

is a surjective local homeomorphism of locales in the sense of [1, Definition A.11]. The family is called a open covering family if each map

$$
U T_{\alpha} \rightarrow U T
$$

is an open embedding. Each of these notions of covering family define a Grothendieck pre-topology on $S$. They both generate the same Grothendieck topology, which we 
shall call the open cover topology on $S$, which is easily seen to be subcanonical. We shall denote by $\operatorname{Sh}(S)$ and $\operatorname{St}(S)$ the category of sheaves on $S$ and the bicategory of stacks on $S$ respectively, both with respect to the open cover topology.

\section{References}

[1] D Carchedi, An étalé space construction for stacks, Alg. Geom. Topol. 13 (2013) 831-903 MR3044595

Department of Mathematical Sciences, George Maison University

4400 University Drive, MS: 3F2, Exploratory Hall, room 4400, Fairfax, VA 22030, USA davidcarchedi@gmail.com

http://math.gmu.edu/ dcarched/

Received: 13 January 2015 Revised: 8 April 2015 